\title{
Hierarchical Routing Protocol for Free-Space Optical Mobile Ad hoc Networks (FSO/RF MANET)
}

\author{
D.Kishore Kumar \\ Assistant Professor, Department of I.T, GITAM \\ Institute of Technology, GITAM University, \\ Visakhapatnam
}

\author{
Y.S.S.R. Murthy \\ Professor \& HOD, Department of CSE \\ Shri Vishnu Engineering College for Women, \\ Vishnupur, \\ Bhimavaram, A.P, India
}

\begin{abstract}
The existing routing protocols in mobile ad hoc network such as DSDV, AODV, DSR, ZRP, SRL, HSR, etc cannot be utilized for FSO/RF MANET owing to certain disjoint characteristics of free space optical (FSO) and MANET. The characteristics include accommodating directionality, accuracy in routing information, memory, reduced overhead and delay. In this paper, a hierarchical routing protocol for FSO/RF MANET is proposed. In this protocol, a network connector which connects the hybrid networks is defined. The cluster formation is based on Neighborhood Discovery Algorithm. In each cluster, the node, which is nearer to the network connector, is elected as cluster head. Each network connector gathers information of all cluster members and their $\mathrm{CH}$ and builds a routing table using network connector discovery algorithm. When the source node of a network wants to send data to destination node in another network, FSO/RF MANET routing protocol is used. Simulation result, show that the proposed routing protocol minimizes the delay while enhancing the end-to-end throughput.
\end{abstract}

\section{Keywords}

Mobile Ad hoc Networks (MANET), Free-Space Optical (FSO), Routing Protocols.

\section{INTRODUCTION}

\subsection{Free-Space Optical (FSO)}

A fibreless, laser-driven technology upholding high bandwidth inclusive of installation connections for last-mile and campus scenarios is termed as Free-Space Optics (FSO). With the help of low powered lasers or LED's, the light pulses are transmitted in a small conical shaped beam through the atmosphere. [1] In order to set up a network link, an optical transceiver is positioned on each side of a transmission path. The transmitter emits a modulated IR signal which is characteristically an infra red (IR) or LED. The high throughput of FSO technology is its main advantage. [2]

\subsubsection{Characteristics of FSO}

The key features of FSO are as follows.

- FSO technology is simple and analogous to fiber optics and makes use of optical transmitters and receivers. However it does not contain fiber cables.

- Though FSO systems can act up to several kilometers distance, there must be adequate power and glimpse among source and destination. [3]

- In FSO, the data can travel in both directions at the same time and hence it is considered as full duplex unit. [1]
- FSO can act as substitute for fiber based MAN solutions. [1]

- The transmitter/ receiver link configurations such as directed, diffuse and hybrid can be attained using FSO unit. [4]

\subsubsection{Applications of FSO}

FSO is concentrated mainly for high altitude application such as space communications and building-top metro-area communications in order to attain high-speed wireless point-topoint communications [5]. The FSO laser systems are extensively utilized in aerospace and defense applications. The fiber optic connections used to connect carriers are deployed by larger corporations. [1]

\subsubsection{Issues of FSO}

FSO has certain issues in spite of offering high throughput which are described as follows.

- $\quad$ The fiber strands cannot be tapped without shattering the strands as they are highly thin which in turn will result in shutdown of the link. [1]

- The main limitations of FSO technology is that it necessitates optical links for upholding line of sight (LOS) [2]

- The climatic changes such as fog and severe weather causes harmful effects on FSO performance.[1]

\subsection{Free-Space Optical MANET}

FSO technology is capable of offering enhanced per-node throughput for mobile ad hoc network (MANET). [4] FSOMANETS can be feasible using "optical antennas". [6] According to the following two principles, FSO structures can be designed in MANET. [5]

- Spatial reprocessing and angular diversity through directional transceivers inlaid on the spherical node surface.

- Auto-alignment circuitry sets up the alignment of two transceivers following a misalignment period.

\subsubsection{FSO/RF MANET Architecture}

FSO/RF MANET can be disintegrated into two subcomponents which are described below.

$\mathbf{1}^{\text {st }}$ subcomponent: It refers to the set of RF based ad hoc networks which is necessary to communicate in real time scenario.

$2^{\text {nd }}$ subcomponent: It facilitates dissemination of information between disjoint ad hoc domains. These are built using hybrid nodes which are members of particular ad hoc network [2] 


\subsubsection{Routing in FSO MANET}

A routing protocol indicates the way by which routers communicate among each other; facilitation of broadcasting information to select routes between any two nodes on a computer network, the route selection performed using routing algorithms. [7]

At the time of routing, FSO topology carries entire traffic in the network. The RF topology is utilized to offer the required backup, as the FSO links are vulnerable to environmental events that include fog, snow, clouds etc. The hybrid nodes help in interconnecting RF and FSO backbone domain space. [2]

A protocol should take features of FSO, RF and MANET into consideration for FSO/RF MANET network. The conventional routing protocol in FSO MANET forwards the unicast packets utilizing directional antenna. But the broadcast packets such as hello packets keep flooding out at every interface to wrap full spread. [9]

\subsection{Problem Identification}

Owing to certain disjoint features of FSO and MANET, the existing routing protocols in MANET cannot be used for FSO MANET. The issues concerned with traditional routing protocol are as follows.

- The routing protocols such as DSDV and AODV make use of reverse path technique but do not take unidirectional links into account [8]. Hence these protocols cannot be directly applied to FSO MANET.

- The protocols namely DSR [12], ZRP [13], or SRL [14] considers unidirectionality by detecting unidirectional links, and subsequently offering a bidirectional abstraction for such links. [8]

- Through probabilistic routing, the routing information decays with time and possess reduced accuracy. [9]

In order to solve routing problems in RF ad-hoc domains, hierarchical state routing (HSR) protocol is used. It is based on a cluster and logical sub network. The source node transmits the data to the destination node through indirect path which results in enhanced delay and overhead. In addition, the scheme for clustering and cluster head selection is not specified.

The protocols such as Neighborhood Discovery Algorithm (NDA) and Base Station Discovery Algorithm (BDA) are utilized to determine routes in FSO- Sensor Networks. This algorithm requires more memory space for discovering neighbor nodes which in turn results in overhead. [8]

To rectify these problems, a hybrid cluster based routing protocol for FSO/RF MANET is proposed in this paper.

\section{RELATED WORK}

Jason Derenick et al. [3] have proposed a Hierarchical link acquisition and routing protocols. Their link acquisition system (LAS) locates robot link partners through high zoom camera systems. Their routing protocol depends on hierarchical state routing (HSR) model suited to FSO/RF paradigm. In real time, they achieved identification using a multi-resolution image representation and normalized intensity distribution (NID). Their technology provides lower per-bit power consumption and a secure/ robust transmission medium. But the effect of link failure and link reacquisition on per-node throughput is not handled.
Bow-Nan Cheng et al. [7] have proposed a Mobile Orthogonal Rendezvous Routing Protocol (MORRP) for mobile ad-hoc networks (MANETs). Their protocol is lightweight and scalable in nature which uses directional communications. They introduced a novel concept termed as directional routing table (DRT) which maps a set-of-IDs to each interface direction to offer probabilistic routing information based on interface direction. Their protocol does not fit into hybrid routing environments.

Unoma Ndili Okorafor et al. [8] have proposed novel neighborhood discovery and base station discovery routing algorithms free-space optical sensor networks (FSOSNs). They considered basic differences in communication pattern between Adhoc and sensor networks. They introduced two novel routing algorithms in FSOSN: The neighborhood discovery algorithm (NDA) is used for local neighborhood discovery and maintenance, while the base station discovery algorithm (BDA) handles uplink and downlink channels discovery.

Abhishek Kashyap et al. [9] have proposed a framework for offering instant backup to traffic in a hybrid RF/FSO mesh network. They provided a framework for modeling FSO link failures and formulate a routing problem to compute backup paths on a given RF topology, for the traffic flowing on FSO topology. Their proposed algorithms possess better performance gain though FSO topology is optimized for the current traffic.

Ziping $\mathrm{Hu}$ et al. [11] have proposed four routing algorithm namely least cost path routing algorithm (LCP), Minimum Hop Count Path Routing Algorithm (MHP), and Minimum Hop Count with Load Balancing Routing Algorithm (MHLB), and Minimum Hop Count Routing Algorithm (MH). They analyzed the performance of these routing protocols in terms of end-toend delay. Their approach results in minimum average delay and least blocked traffic.

\section{PROPOSED SOLUTION}

\subsection{Overview}

In this paper, a hybrid cluster based routing protocol for FSO/RF MANET is proposed. In this technique, a network connector is defined which connects the hybrid networks. The clusters are formed using Neighborhood Discovery Algorithm. In each cluster, the node, which is nearer to the network connector, is elected as cluster head. Each network connector gathers information of all cluster members and their $\mathrm{CH}$ and builds a routing table using network connector discovery algorithm. When the source node of a network wants to send data to destination node in another network, FSO/RF MANET routing protocol is used.

\subsection{Neighbor Discovery Algorithm (NDA)}

The process for a node to identify all its neighbors is called neighbor discovery. Neighbor discovery algorithms can be classified into two categories.

Randomized neighbor discovery: In randomized neighbor discovery, each node transmits at randomly chosen times and discovers all its neighbors by a given time with high probability.

Deterministic neighbor discovery: In deterministic neighbor discovery, on the other hand, each node transmits according to a predetermined transmission schedule that allows it to discover all its neighbors by a given time with probability one.

In our technique, we use randomized neighbor discovery algorithm for cluster formation phase. (Shown in section 3.3.1) 


\subsection{Proposed Technique}

The proposed technique consists of three phases namely.

- Cluster Formation,

- Network Connector Discovery

- FSO/RF MANET Routing.

\subsubsection{Phase 1-Cluster Formation}

Let $\mathrm{N}_{\mathrm{i}}$ represent the nodes deployed in the network. (where $\mathrm{i}=$ $1,2,3, \ldots . ., \mathrm{n})$

Let $\mathrm{N}_{\text {nei }}$ represents the neighbor nodes.

Let $\mathrm{C}_{\mathrm{i}}$ represents the clusters

Let $\mathrm{CH}_{\mathrm{i}}$ be the cluster head.

Let $n\left(C_{i}\right)$ be the number of nodes in the cluster

Let $\mathrm{NC}_{\mathrm{i}}$ represent the network connectors that connects hybrid networks and it has virtual link with all cluster member via CHs.

Let Th represents the threshold.

Also we assume that each node maintains a neighbor table that includes the ID of the neighboring nodes and link status.

The steps involved in cluster formation are as follows.

\section{Step 1}

Each $\mathrm{NC}_{\mathrm{i}}$ broadcast HELLO message to all $\mathrm{N}_{\text {nei }}$ using NDA.

$$
\mathrm{N}_{\mathrm{i}} \stackrel{\text { Hello }}{\longrightarrow} \mathrm{N}_{\text {nei }}
$$

The hello message will include the sender nodes ID, sender nodes status (say energy) and neighboring node status. The format of Hello message is as follows.

Table 1. Format of Hello Message

\begin{tabular}{|l|l|l|l|}
\hline $\begin{array}{l}\text { Sender node } \\
\text { ID }\end{array}$ & Node status & \multicolumn{2}{|l|}{$\begin{array}{l}\text { Neighboring } \\
\text { Table }\end{array}$} \\
\hline & & $\mathrm{N}_{\text {nei }}$ & $\begin{array}{l}\text { Node } \\
\text { status }\end{array}$ \\
\hline
\end{tabular}

Step 2

When any $\mathrm{N}_{\mathrm{ei}}$ receives the HELLO message, it verifies whether the message possess its ID using the following condition.

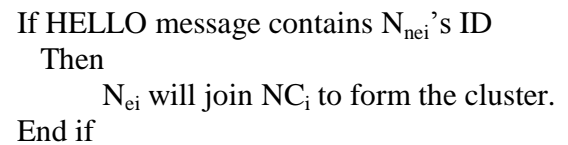

Step 3

The process in step 2 is iterated pre-defined threshold of nodes joins the cluster.

If $n\left(C_{i}\right)=T h$

Then

End if

Pause step 2.

\section{Step 4}

When any $\mathrm{N}_{\text {nei }}$ receives more than one HELLO message, it will join with nearer $\mathrm{NC}_{\mathrm{i}}$ with maximum energy. Currently, the hybrid network comprises of many clusters.

\section{Step 5}

In each cluster, the node, which is nearer to $\mathrm{NC}_{\mathrm{i}}$ can be elected as cluster head $\left(\mathrm{CH}_{\mathrm{i}}\right)$.

Fig. 1 demonstrates the broadcast of HELLO message by the network connectors. The network connector node $\mathrm{NC}_{3}$ broadcast the HELLO message to its neighbor nodes $\mathrm{N}_{\text {ne1 }}, \mathrm{N}_{\mathrm{ne} 2}$, $\mathrm{N}_{\mathrm{ne} 4}, \mathrm{~N}_{\mathrm{ne} 5}, \mathrm{~N}_{\mathrm{ne} 7}$, and $\mathrm{N}_{\mathrm{ne} 10}$. Similarly $\mathrm{NC}_{8}, \mathrm{NC}_{11}, \mathrm{NC}_{13}$ and $\mathrm{NC}_{14}$ broadcast the HELLO message to its neighbor nodes $\left(\mathrm{N}_{\text {ne5 }}\right.$, $\mathrm{N}_{\text {ne7 }}, \mathrm{N}_{\mathrm{ne} 9}, \mathrm{~N}_{\mathrm{ne} 11}, \mathrm{~N}_{\mathrm{ne} 13}$, and $\left.\mathrm{N}_{\mathrm{ne} 15}\right),\left(\mathrm{N}_{\mathrm{ne} 7}, \mathrm{~N}_{\mathrm{ne} 12}, \mathrm{~N}_{\mathrm{ne} 13}\right.$, and $\left.\mathrm{N}_{\text {ne14 }}\right),\left(\mathrm{N}_{\mathrm{ne} 8}, \mathrm{~N}_{\mathrm{ne} 11}, \mathrm{~N}_{\mathrm{ne} 14}, \mathrm{~N}_{\mathrm{ne15}}, \mathrm{N}_{\mathrm{ne} 16}\right.$, and $\left.\mathrm{N}_{\mathrm{ne} 17}\right)$ and $\left(\mathrm{N}_{\text {ne12 }}\right.$, $\mathrm{N}_{\mathrm{ne} 13}, \mathrm{~N}_{\mathrm{ne} 17}, \mathrm{~N}_{\mathrm{ne} 18}, \mathrm{~N}_{\mathrm{ne} 19}$, and $\mathrm{N}_{\mathrm{ne} 20}$ ).

Fig 2 shows the formation of clusters $\mathrm{C}_{1}, \mathrm{C}_{2}, \mathrm{C}_{3}$ and $\mathrm{C}_{4}$. The nodes $\mathrm{N}_{1}, \mathrm{~N}_{13}, \mathrm{~N}_{15}$, and $\mathrm{N}_{19}$ which are near to $\mathrm{NC}_{3}, \mathrm{NC}_{11}, \mathrm{NC}_{8}$, and $\mathrm{NC}_{14}$ are selected as $\mathrm{CHs}$.
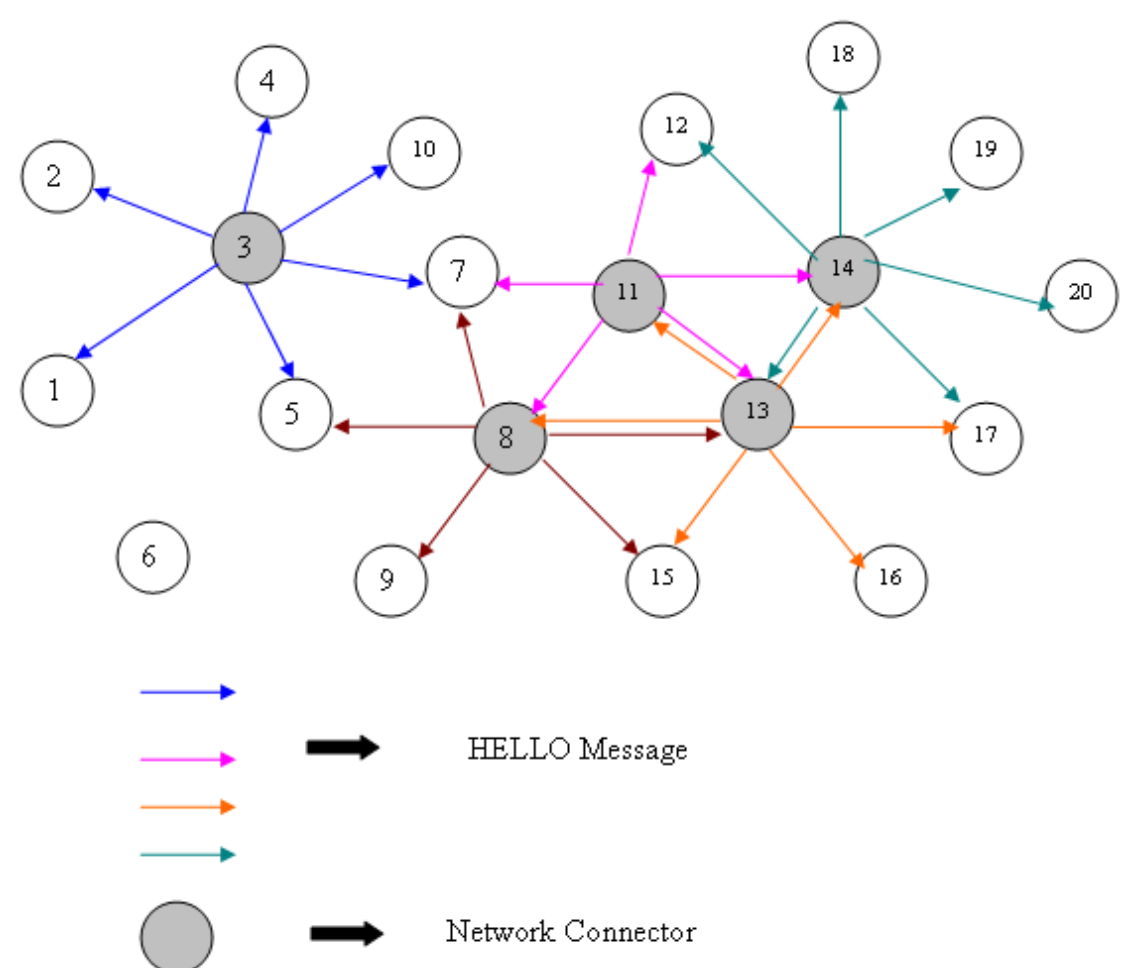

Fig 1 Broadcast of Hello messages 

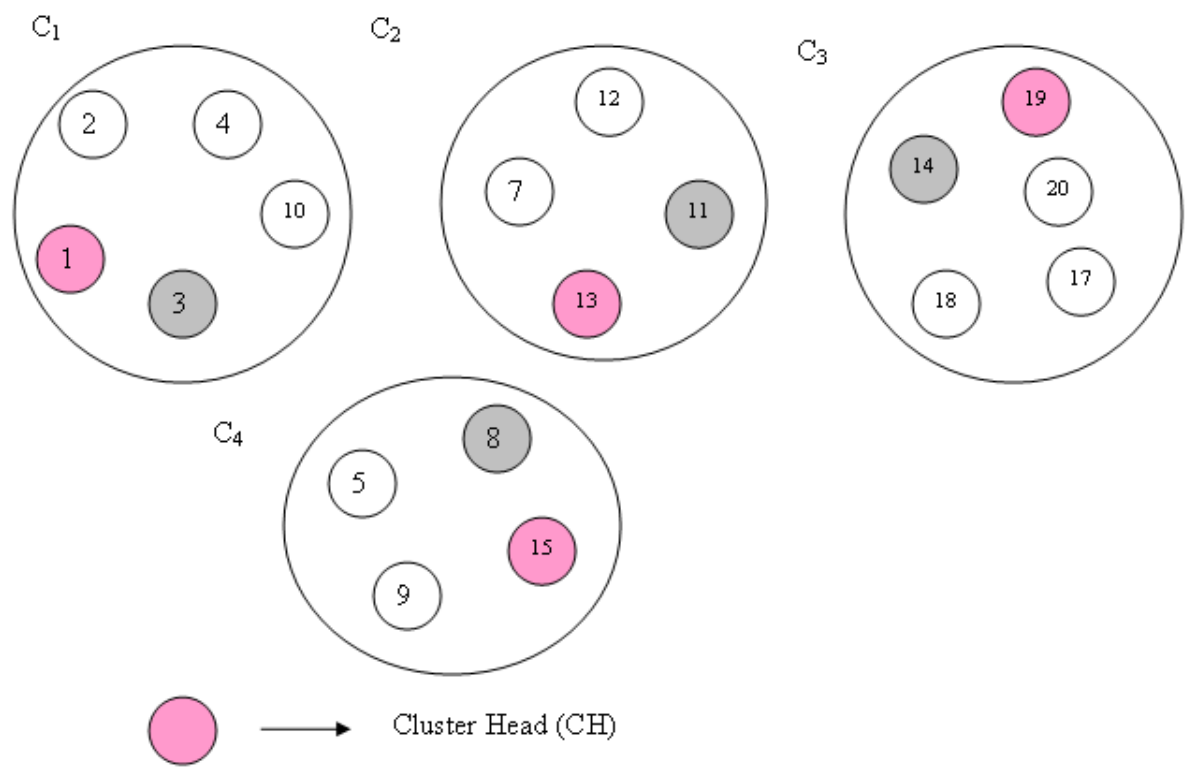

Fig 2 Cluster Formation

\subsubsection{Phase 2-Network Connector Discovery} Algorithm (NCDA)

Let ID (NC) represent the identity of NC.

Let $\mathrm{Sq}(\mathrm{ID})$ represents the sequence number of $\mathrm{NC}$.

Let $\mathrm{CM}_{\mathrm{i}}$ be the cluster members

Let $\mathrm{CH}_{\mathrm{i}}$ be the cluster heads.

Consider that there are $n$ clusters $\left\{\mathrm{C}_{1}, \mathrm{C}_{2}, \mathrm{C}_{3} \ldots, \mathrm{C}_{\mathrm{n}}\right\}$. Each NC should contain the routing table that includes the information about all the cluster members and their respective cluster heads. The routing table is constructed with the help of network connection discovery algorithm (NCDA). The steps involved in NCDA are as follows.

\section{Step 1}

Each $\mathrm{NC}_{\mathrm{i}}$ sends network connector discovery (NCD) message to all other $\mathrm{NC}_{\mathrm{i}}$ in the network through $\mathrm{CH}_{\mathrm{i}}$.

The format of NCD message is as follows.

Table 2. Format of NCD message

\begin{tabular}{|l|l|l|l|}
\hline $\mathrm{D}(\mathrm{NC})$ & $\mathrm{Sq}(\mathrm{NC})$ & $\mathrm{CM}_{\mathrm{i}}$ & $\mathrm{CH}_{\mathrm{i}}$ \\
\hline
\end{tabular}

\section{Step 2}

Any $\mathrm{NC}_{\mathrm{i}}$ upon receiving NCD message builds its routing table with the information in NCD.

\section{Step 3}

The process involved in step 1 and 2 is iterated until $\mathrm{NC}_{\mathrm{i}}$ receives NCD message from $\mathrm{CH}_{n}$ i.e. last $\mathrm{CH}$.

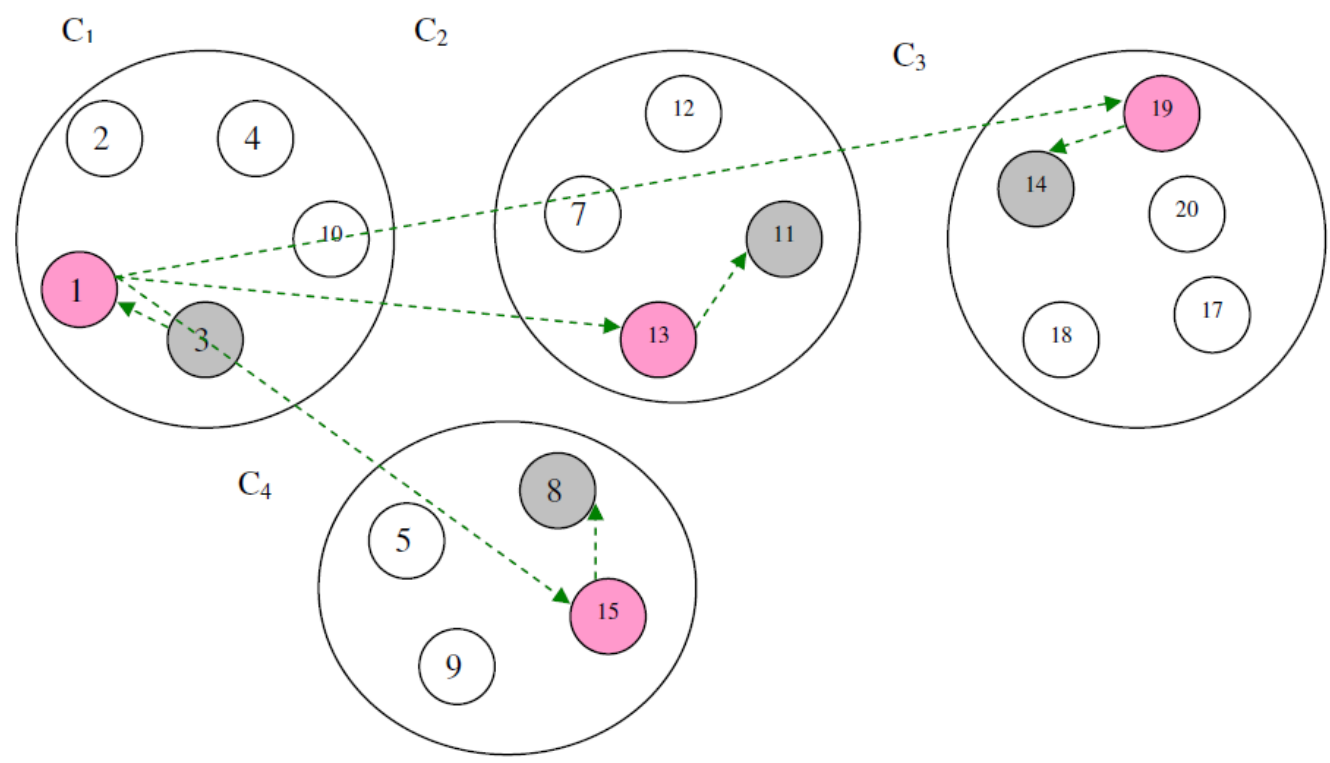

Fig 3 Neighbor Connector Discovery 
Consider Fig. 3. $\mathrm{NC}_{3}$ transmits $\mathrm{NCD}$ message to its $\mathrm{CH}_{1}$. $\mathrm{CH}_{1}$ broadcast the NCD message to $\mathrm{CH}_{13}, \mathrm{CH}_{15}$ and $\mathrm{CH}_{19}$. These $\mathrm{CHs}$ transmits its $\mathrm{NCD}$ to its $\mathrm{NC}$ such as $\mathrm{NC}_{11}, \mathrm{NC}_{8}$ and $\mathrm{NC}_{14}$ respectively. All these network connectors build its routing table with information about the $\mathrm{C}_{1}$ obtained from the NCD message. This process is iterated until $\mathrm{NC}_{3}$ receives $\mathrm{NCD}$ message from last $\mathrm{CH}$.

\subsubsection{FSO/RF MANET Routing}

Let $\mathrm{S}$ and $\mathrm{D}$ be source and destination node respectively.

Let $\mathrm{ID}_{\mathrm{D}}$ be the identity of destination node

Let $\Psi$ represent the set of $\mathrm{NC}_{\mathrm{i}}$ in the hybrid network.

Let DNC be the destination NC.

When $S$ wants to transmit the data to $\mathrm{D}$ in another network, $\mathrm{S}$ initially transmits the data along with the D's identity $\left(\mathrm{ID}_{\mathrm{D}}\right)$ to its respective $\mathrm{NC}_{\mathrm{i}}$ via its $\mathrm{CH}_{\mathrm{i}}$. This $\mathrm{NC}_{\mathrm{i}}$ is considered as home $\mathrm{NC}(\mathrm{HNC})$

$$
\mathrm{S} \stackrel{\left[I D_{D}\right][D A T A]}{\longrightarrow} \mathrm{CH}_{\mathrm{i}} \stackrel{\left[I D_{D}\right][D A T A]}{\longrightarrow} \mathrm{HNC}
$$

$\mathrm{HNC}$ verifies its local routing table for $\mathrm{ID}_{\mathrm{D}}$.

$$
\begin{aligned}
& \text { If } \mathrm{HNC} \text { does not contain } \mathrm{ID}_{\mathrm{D}} \\
& \text { Then } \\
& \mathrm{HNC} \stackrel{\left[R E Q\left(I D_{D}\right)\right]}{\longrightarrow} \Psi \\
& \text { End if } \\
& \text { If any } \mathrm{NC}_{\mathrm{i}} \text { within } \Psi \text { contains } \mathrm{ID}_{\mathrm{D}} \\
& \text { Then } \\
& \text { Particular } \mathrm{NC}_{\mathrm{i}} \text { becomes DNC } \\
& \mathrm{DNC} \stackrel{[\operatorname{Inf} o(D)]}{\longrightarrow} \mathrm{HNC} \\
& \mathrm{HNC} \stackrel{[\text { data }]}{\longrightarrow} \mathrm{DNC} \stackrel{[\text { data }]}{\longrightarrow} \mathrm{D}
\end{aligned}
$$

The NC first checks in the local routing table for the destination node id. If it is not found, broadcast a request to other NCs. The $\mathrm{NC}$ which contains the corresponding destination node, send the details to the home NC. The home NC then sends the data to the destination $\mathrm{NC}$, which in turn sends it to the destination node by using its routing table information.

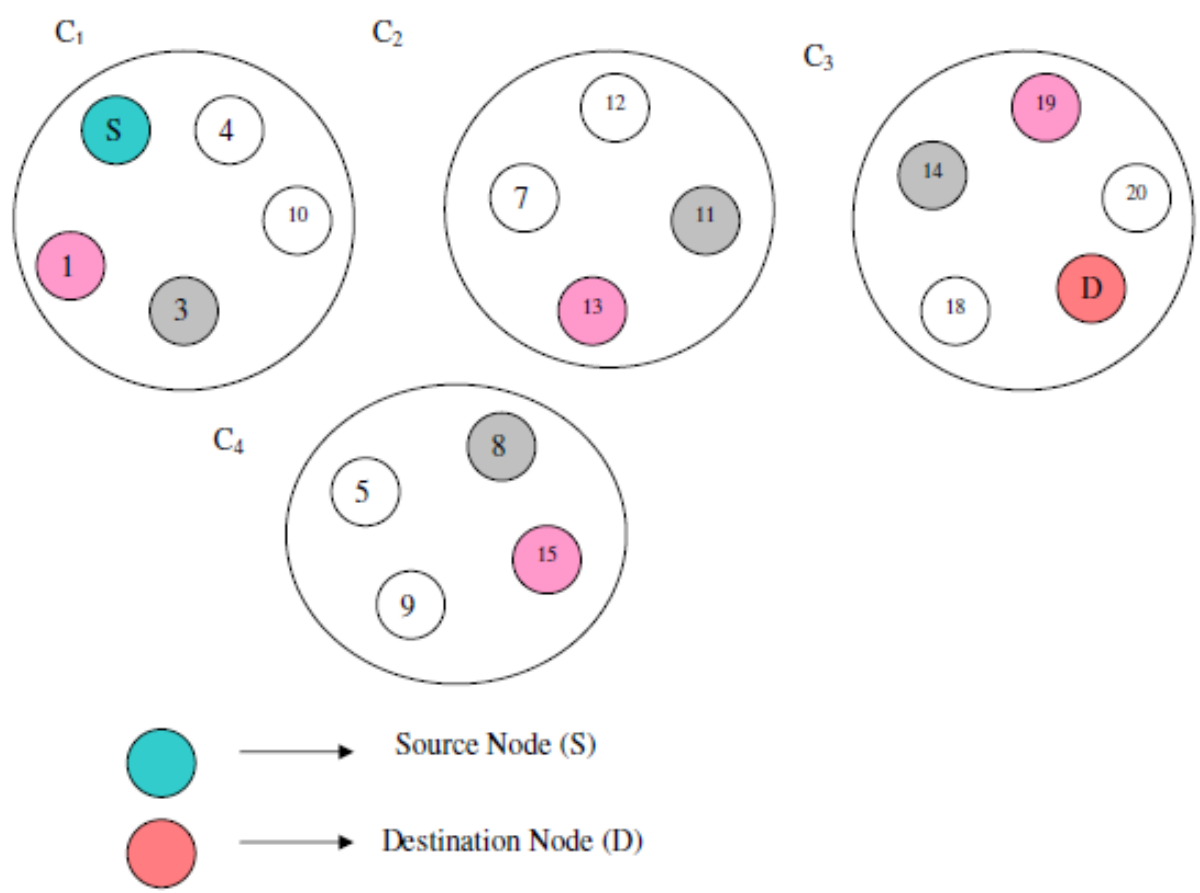

Fig 4 demonstrates the routing in FSO/RF mobile ad hoc network.

When $\mathrm{S}$ wants to transmit the data to $\mathrm{D}$ in another network, $\mathrm{S}$ initially transmits the data along with the D's identity $\left(\mathrm{ID}_{\mathrm{D}}\right)$ to its respective $\mathrm{NC}_{3}(\mathrm{HNC})$ via its $\mathrm{CH}_{1} \cdot \mathrm{NC}_{3}$ verifies its local routing table for $\mathrm{ID}_{\mathrm{D}}$. If $\mathrm{NC}_{3}$ does not contain $\mathrm{ID}_{\mathrm{D}}$, it broadcasts a destination request to $\Psi . \mathrm{NC}_{14}$ contains $\mathrm{D}$ and it forwards the information about $\mathrm{D}$ to $\mathrm{NC}_{3}$. $\mathrm{NC}_{3}$ sends the data to $\mathrm{NC}_{14}$ which in turn is send to $\mathrm{D}$.

\section{SIMULATION RESULTS}

\subsection{Simulation Setup}

The performance of our Hierarchical Routing Protocol (HRP) is evaluated through NS2 [15] simulation. A random network deployed in an area of $500 \times 500 \mathrm{~m}$ is considered. The number of nodes is varied as $25,50,75$ and 100 . Initially the nodes are placed randomly in the specified area. In the simulation, the channel capacity of mobile hosts is set to the same value: 2 Mbps. The distributed coordination function (DCF) of IEEE
802.11 is used for wireless LANs as the MAC layer protocol. The simulated traffic is CBR with UDP source and sink.

Table 3 summarizes the simulation parameters used.

Table 3: Simulation Parameters

\begin{tabular}{|l|l|}
\hline No. of Nodes & $25,50,75$ and 100 \\
\hline Area Size & 2200 X 2200 \\
\hline Mac & 802.11 \\
\hline Simulation Time & $50 \mathrm{sec}$ \\
\hline Traffic Source & CBR \\
\hline Packet Size & 512 \\
\hline Transmission Range & $75 \mathrm{~m}$ \\
\hline Rate & $250 \mathrm{~Kb}$. \\
\hline Propagation Model & Free Space Optical \\
\hline Antenna Type & FSOAntenna \\
\hline Modulation Type & BPSK/FSO \\
\hline
\end{tabular}




\subsection{Performance Metrics}

The performance of HRP is compared with non cluster based AODV protocol. The performance is evaluated mainly, according to the following metrics

Average end-to-end Delay: The end-to-end-delay is averaged over all surviving data packets from the sources to the destinations.

Average Packet Delivery Ratio: It is the ratio of the number .of packets received successfully and the total number of packets transmitted.

Drop: It is the number of packets dropped during the data transmission.

Throughput: It is the total number of packets received by the receiver.

\subsection{Results}

\section{A. Based on Nodes}

In our initial experiment the number of nodes is varied as 25 , 50,75 and 100.

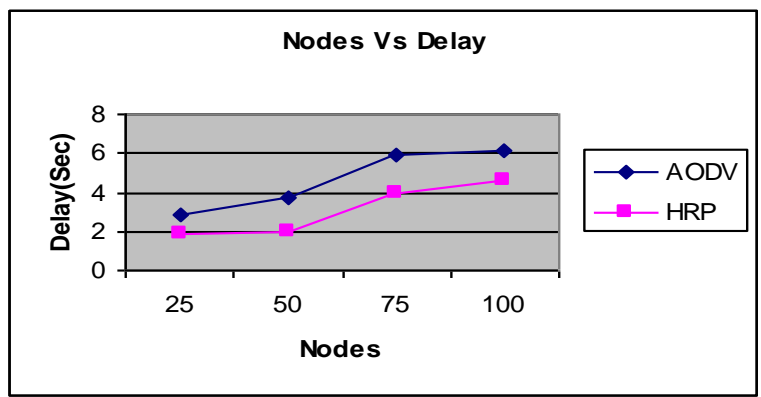

Fig 5: Nodes Vs Delay

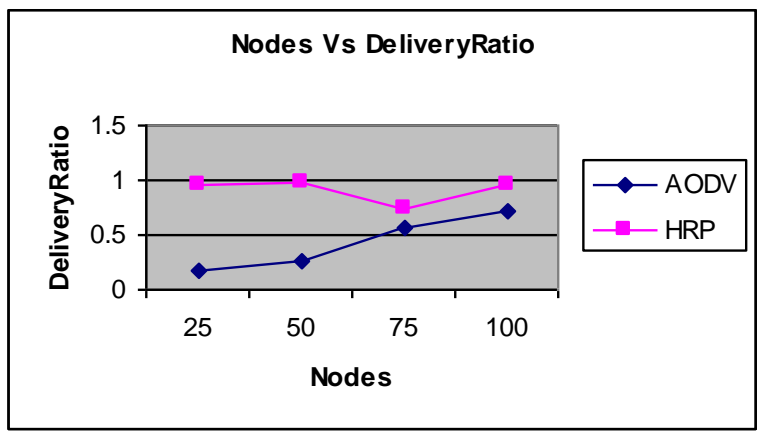

Fig 6: Nodes Vs Delivery Ratio

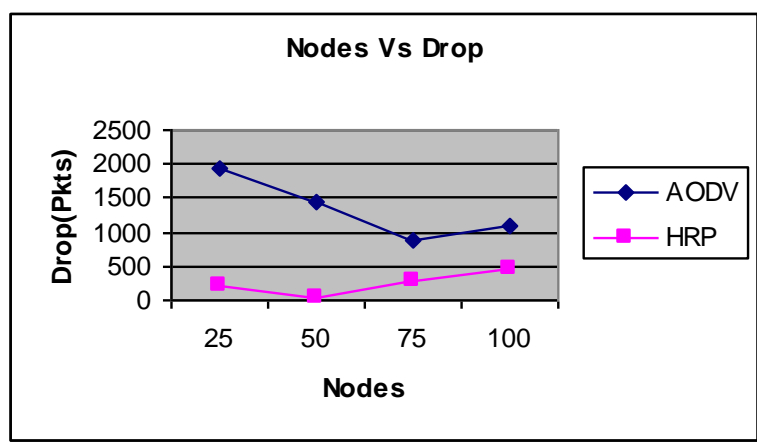

Fig 7: Nodes Vs Drop

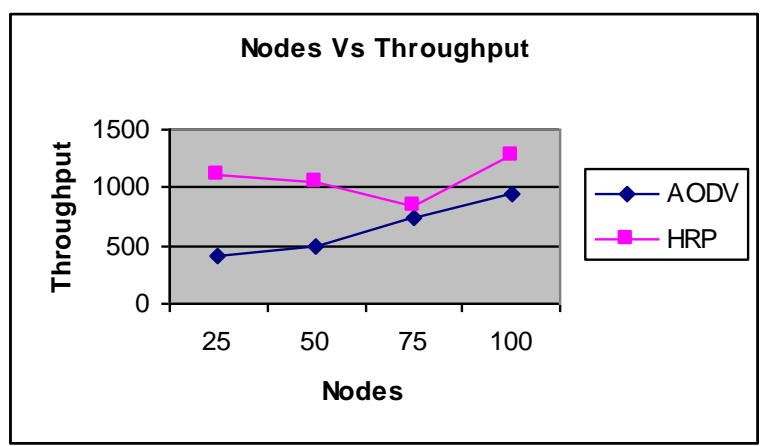

Fig 8: Nodes Vs Throughput

Fig. 5 shows the delay occurred for both the protocols. When the number of nodes is increased, the delay is also increased. From the figure, it can be seen that the end-to-end delay of the proposed HRP is less than AODV, because of the hierarchical routing. From Fig. 7, the packet drop is less for HRP when compared to AODV. So the throughput and packet delivery ratio is high for HRP, as depicted in figures 8 and 6 , respectively.

\section{CONCLUSION}

In this paper, a hierarchical routing protocol for $\mathrm{FSO} / \mathrm{RF}$ MANET is proposed. The cluster formation is based on Neighborhood Discovery Algorithm. In each cluster, the node, which is nearer to the network connector, is elected as cluster head. Each network connector gathers information of all cluster members and their $\mathrm{CH}$ and builds a routing table using network connector discovery algorithm. When the source node of a network wants to send data to destination node in another network, FSO/RF MANET routing protocol is used. By simulation result, it has been shown that the proposed routing protocol minimizes the delay and overhead, while enhancing the end-to-end throughput

\section{REFERENCES}

[1] Sans Institute Infosec, "Free-Space Optics: A Viable, Secure Last-Mile Solution?" www.sans.org/.../free-spaceoptics-viable-secure-last-mil.

[2] Mehmet Bilgi and Murat Yuksel, "Multi-Element FreeSpace-Optical Spherical Structures with Intermittent Connectivity Patterns" IEEE Infocom, 2008.

[3] Jason Derenick, Christopher Thorne and John Spletzer, "On the Deployment of a Hybrid Free-space Optic/Radio Frequency (FSO/RF) Mobile Ad-hoc Network", IEEE Intelligent Robot and Systems, 2005.

[4] Amjad Umar, "Ten Emerging Wireless Networks: UWB, FSO, MANET and Flash OFDM", www.amjadumar.com.

[5] Behrooz Nakhkoob, Mehmet Bilgi, Murat Yuksel, and Mona Hella, "Multi Transceiver Optical Wireless Spherical Structures for MANETs", IEEE Journals on Selected Areas in Communications, 2009.

[6] Jason Derenick, Christopher Thorne and John Spletzer, "Hybrid Free-Space Optics/Radio Frequency (FSO/RF) Networks for Mobile Robot Teams", Multi Robot Systems, 2005.

[7] Bow-Nan Cheng, Murat Yuksel, Shivkumar and Kalyanaraman, "Using Directionality in Mobile Routing", IEEE International Conference on Mobile Adhoc and Sensor Systems, 2008. 
[8] Unoma Ndili Okorafor and Deepa Kundur, "Efficient Routing Protocols for a Free Space Optical Sensor Network", IEEE International Conference on Mobile Adhoc and Sensor Network, 2005.

[9] Abhishek Kashyap, Anuj Rawat and Mark Shayman, "Integrated Backup Topology Control and Routing of Obscured Traffic in Hybrid RF/FSO Networks", IEEE Globe COM, 2006.

[10] www.wikipedia.org

[11] Ziping Hu, Pramode Verma, and James Sluss Jr, "Routing in Degree-constrained FSO Mesh Networks", International Journal of Hybrid Information Technology, 2009.
[12] D. B. Johnson and D. A. Maltz, "Dynamic source routing in ad hoc wireless networks," Mobile Computing, vol. 353, Kluwer Academic Publishers, 1996.

[13] M. R. Pearlman Z. J. Haas and P. Samar, "The zone routing protocol (zrp) for ad hoc networks," Internet Draft - Mobile Ad Hoc Networking (MANET) Working Group of the Internet Engineering Task Force (IETF), 2001.

[14] V. Ramasubramanian, R. Chandra, and D. Mosse, "Providing a bidirectional abstraction for unidirectional ad hoc networks," IEEE Infocom, pp. 1258-1267, 2002.

[15] Network Simulator: http:///www.isi.edu/nsnam/ns 